MATHEMATICS OF COMPUTATION

Volume 80, Number 274, April 2011, Pages 1019-1036

S $0025-5718(2010) 02431-7$

Article electronically published on October 5, 2010

\title{
DILATIONAL INTERPOLATORY INEQUALITIES
}

\author{
MARKUS HEGLAND AND ROBERT S. ANDERSSEN
}

\begin{abstract}
Operationally, index functions of variable Hilbert scales can be viewed as generators for families of spaces and norms and, thereby, associated scales of interpolatory inequalities. Using one-parameter families of index functions based on the dilations of given index functions, new classes of interpolatory inequalities, dilational interpolatory inequalities (DII), are constructed. They have ordinary Hilbert scales (OHS) interpolatory inequalities as special cases. They represent a precise and concise subset of variable Hilbert scales interpolatory inequalities appropriate for deriving error estimates for peak sharpening deconvolution. Only for Gaussian and Lorentzian deconvolution do the DIIs take the standard form of OHS interpolatory inequalities. For other types of deconvolution, such as a Voigt, which is the convolution of a Gaussian with a Lorentzian, the DIIs yield a new class of interpolatory inequality. An analysis of deconvolution peak sharpening is used to illustrate the role of DIIs in deriving appropriate error estimates.
\end{abstract}

\section{INTRODUCTION}

In the analysis of the numerical performance of traditional regularization methods (Engl et al. [5]), interpolatory inequalities between the norms $\|u\|_{a}, a \in R_{+}$, generated by an appropriate scale (family) of Hilbert spaces $H_{a}=$ Domain $\left(T^{a / 2}\right)$, play the central role. In terms of the original concept of a Hilbert scale, as introduced by Krein and Petunin [12] and generated by a densely-defined, unbounded, self-adjoint and strictly positive operator $T$, such inequalities take the form

$$
\|u\|_{\theta r+(1-\theta) s} \leq\|u\|_{r}^{\theta}\|u\|_{s}^{1-\theta}, \quad r<s, \quad 0 \leq \theta \leq 1,
$$

where $\|u\|_{a}=\left\|T^{a / 2} u\right\|$. Through the appropriate choice of $T$ and the values for $r$ and $s$, the corresponding inequality (11) can be used to derive estimates for the error $e$ of the regularized solution of improperly posed operator equations that simultaneously take account of both the compact and smoothing nature of the operator (Groetsch [7, Natterer [17, Schröter and Tautenhahn [21, Tautenhahn 22]). Typically, such inequalities lead to bounds for the error $e$ of the form $\|e\| \leq C \delta^{\theta}$, where $\delta$ is a measure of the error in the data.

When utilizing such inequalities to derive error estimates for linear improperly posed problems, it was observed by various authors that realistic error estimates could only be derived for a subset of linear improperly posed operator equations. This led to the need to construct more general counterparts of the inequality (1). Hegland [8, 9], by exploiting the spectral decomposition of an appropriately chosen operator $T$, first introduced the concept of a variable Hilbert scale (VHS) for a quite

Received by the editor March 18, 2008 and, in revised form, March 9, 2010.

2010 Mathematics Subject Classification. Primary 65J20.

(C) 2010 CSIRO, Mathematics, Informatics and Statistics 
general index function $\phi$. Then, by invoking appropriate regularity about the choice of the index function, Hegland established how more general counterparts of the interpolatory inequality (1) could be constructed.

The utility of this basic concept of VHS, in deriving more representative interpolatory inequalities, error estimates and convergence rates for the regularized solution of improperly posed operator equations, has been subsequently exploited and/or modified by various authors including Nair et al. [16], Mathé and Pereverzev [13, 14, Mathé and Tautenhahn [15] and Bégout and Soria 3.

VHS interpolatory inequalities not only overcome the mentioned shortcoming of interpolatory inequalities of the form (11), but also allow a greater variety of interpolatory inequalities to be constructed. It is this aspect that is pursued in this paper. A recent example can be found in Nair et al. 16. In our paper, the VHS framework and associated index functions are introduced using the spectral theorem for positive definite self-adjoint operators. The special subclass of dilational Hilbert scales (DHS) is then defined, and new families of interpolatory inequalities are derived and applied.

The motivation is the analysis of deconvolution peak sharpening (Hegland and Anderssen [11]). For a given peak function $b(t) \in L_{2}(\mathbb{R})$, its corresponding dilations will be denoted by

$$
b_{\gamma}(t)=\frac{1}{\gamma} b(t / \gamma), \quad 0<\gamma<\infty,
$$

and the associated convolution operators by

$$
B_{\gamma} f:=b_{\gamma} * f:=\int_{-\infty}^{\infty} b_{\gamma}(x-y) f(y) d y .
$$

In the sequel, the scaling $\gamma$ is chosen so that $\gamma=1$ defines the observational process that is performed in applications, such as various forms of spectroscopy (as discussed in Section 3). Consequently, $B$ models, via the equations

$$
u=B f, \quad B=B_{1},
$$

the convolutional broadening that the measurement process has imposed on the exact spectrum $f$ to give the observed signal $u$.

When $b(t)$ is the Gaussian peak

$$
g(t)=\exp \left(-t^{2} / 2\right)
$$

the convolutional relationship

$$
g=g_{\alpha} * g_{\beta}, \quad 1=\alpha^{2}+\beta^{2},
$$

can be used to factor

$$
u=g * f=G f
$$

in the following manner

$$
u=G_{\alpha} G_{\beta} f=G_{\alpha} z, \quad z=G_{\beta} f
$$

with $\alpha$ determining the proportion of the full broadening to be deconvolved and $\beta$ the maximum extent of the broadening that the resulting solutions will have. From an interpolatory inequality perspective, $\beta$ represents the characterization of the source condition. In this way, $\alpha$ and $\beta$ perform a trade-off between the amount of deconvolution to be performed and the achievable rates of convergence, as outlined in Section 3. 
Though, in general, such an explicit decomposition does not hold for nonGaussian and non-Lorentzian peak broadening, it can be adapted for dilational models, with peak functions $b(t)$. An important example is the Voigt peak [18], which is formed by convolving a Gaussian peak with a Lorentzian. The adaptation is achieved by performing the decomposition

$$
u=B f=B B_{\beta}^{-1} B_{\beta} f=\tilde{B}_{\beta} z, \quad \tilde{B}_{\beta}=B B_{\beta}^{-1}, \quad z=B_{\beta} f, \quad 0<\beta<1,
$$

where the operator $\tilde{B}_{\beta}$ is well defined on the $H_{\beta}:=\operatorname{Range}\left(B_{\beta}\right)$. Thus, $\tilde{B}_{\beta}$ and $\tilde{B}_{\beta} z=u$ correspond, respectively, to the "sharpening operator" and "sharpening equation", while any regularization method used to solve it will be referred to as a "sharpening procedure". Since the exact solution $z \in H_{\beta}$, this can be viewed as the source condition which controls the achievable rates for a given regularization methodology. A discussion about how $z=B_{\beta} f$ provides an appropriate source condition is given in Section 3. For example, for spectral sharpening with known peak function $b(t)$, interpolation inequalities of the following form are required:

$$
\left\|B_{\beta} v\right\| \leq F(\|v\|,\|B v\|), \quad v \in L_{2}(\mathbb{R})
$$

where the structure of the function $F$ highlights the trade-off between $\|v\|$ and $\|B v\|$.

It is shown in the sequel that, in particular, one has, in many situations, relationships of the form

$$
\left\|B_{\beta} v\right\| \leq \frac{\|v\|}{a\left(\beta a^{-1}\left(\|v\|^{2} /\|B v\|^{2}\right)\right)^{1 / 2}}
$$

where $a(t)=1 /|\hat{b}(t)|^{2}$, and $\hat{b}$ denotes the Fourier transform of $b$. In fact, this inequality recovers the standard interpolation inequalities for both Gaussian and Lorentzian peaks.

An application of this inequality arises when $b(t)$ is the convolution of a Gaussian with a Lorentzian peak, the Voigt peak (with $\hat{b}(t)=\exp \left(-t^{2} / 2\right) \exp (-\mu t / 2)$ ), which is common in applications [18, yields

$$
\left\|B_{\beta} v\right\| \leq\|B v\|^{\zeta(v)}\|v\|^{1-\zeta(v)}
$$

where

for some $\|B v\| /\|v\| \leq \epsilon \leq 1$.

$$
\zeta(v)=\beta^{2}+\frac{\mu(1-\beta) \beta}{(1+\mu /(2|\log (\epsilon)|))},
$$

For the exponential peak with $b(\nu)=\exp (-|\nu|)$, we derive the new interpolation inequality

$$
\left\|B_{\beta} v\right\| \leq \frac{\|B v\|\|v\|}{\left(\beta^{2}\|v\|+\left(1-\beta^{2}\right)\|B v\|\right)} .
$$

By deriving various lower bounds for the denominator in this last equation, the following upper bounds can be determined, which hold for all $\beta$,

$$
\left\|B_{\beta} v\right\| \leq \begin{cases}(a) & \beta^{-2}\|B v\| \\ (b) & \|B v\|^{\beta^{2}}\|v\|^{1-\beta^{2}}\end{cases}
$$

These bounds illustrate some interesting features about exponential peak deconvolution. The first bound $(a)$ illustrates that exponential peak deconvolution is properly posed as the norm of the reconstruction error $\left\|B_{\beta} v\right\|$ is proportional to 
the norm of the observational error $\|B v\|$. In a way, it is a reflection of the cusp in the exponential peak at the origin. It is a useful fact when $\beta \sim 1$, as little deconvolution is being performed. On the other hand, as $\beta \rightarrow 0$, the deconvolution becomes strongly ill-conditioned. Now, the bound $(b)$ gives the more appropriate assessment of the situation.

The paper has been organized in the following manner. A very general Hilbert scale (VHS) inequality is proved in Section 2 along with a general Hölder inequality. The Hilbert scales based on dilations of index functions are then introduced. Their interpolation inequalities (DIIs) are then derived from the general VHS interpolation inequality. Both cases of increasing index functions (leading to "positive norms") and decreasing index functions (leading to "negative norms") are considered. In Section 3, the DIIs are applied to derive error bounds for peak sharpening procedures. Initially, various bounds and results for general peaks are derived. The error bounds for the sharpening of Gaussian, Lorentzian, Voigt and exponential peaks are then given. The bounds for the Gaussian and Lorentzian peaks can also be obtained from the classical Hilbert scale theory and are confirmed by the DII theory. The error bounds for Voigt and exponential peaks, however, cannot be obtained using the classical theory. Consequently, a new framework and theory is required. It turns out that the best error bounds are derived when using the negative norms and by interpreting the sharpening procedure as a mollification process. Conclusions are given in Section 4 along with a brief overview of some open problems.

\section{VARiable AND Dilational Hilbert scales}

The spectral theorem for a positive definite selfadjoint operator $T$ on a Hilbert space $H$ (see, e.g., 20]) induces (for each $T$ ) a family $E(\lambda)$ of orthogonal projections such that $E_{f, g}(\lambda):=(E(\lambda) f, g)$ defines a Stieltjes measure on $(0, \infty)$ and

$$
(T f, g)=\int_{0}^{\infty} \lambda d E_{f, g}(\lambda)
$$

for all $g \in H$ and $f \in D(T)$ (the domain of $T$ ). One can see that $(E(\lambda) f, g)$ is right semi-continuous. In applications, the operator $T$ is often a differential operator like the Laplacian. Intuitively, this representation generalizes the concept of an eigenvalue decomposition of a real symmetric matrix.

Following the definition in [9, let any continuous function $\phi:(0, \infty) \rightarrow(0, \infty)$ be called an index function. Then

$$
(f, g)_{\phi}:=\int_{0}^{\infty} \phi(\lambda) d E_{f, g}(\lambda), \quad\|f\|_{\phi}^{2}=(f, f)_{\phi}
$$

is a densely defined bilinear form on $H$ with scalar product $(\cdot, \cdot)_{\phi}$. Let the closure of the domain of this bilinear form be denoted $H_{\phi}$. Note that $H_{\phi}$ then becomes a Hilbert space with scalar product $(\cdot, \cdot)_{\phi}$ and we call the set of all possible $H_{\phi} a$ variable Hilbert scale.

In [3, Bégout and Soria introduce a generalization of the Hölder inequality for $L^{p}$ spaces. It turns out that this inequality is closely connected to the "variable Hilbert scale" inequality introduced by Hegland in [9]. There are two main differences between the two results: First, the variable Hilbert scale inequality uses the spectral measure to define the underlying norms, while the Hölder inequalities are based on the Lebesgue measure; second, the two inequalities are slightly different in their 
conditions and bounds. In the next theorem, we give both results together with a proof which is an adaptation of the proof in [3]. While the first result could be obtained by replacing the $f$ of [3] by 1 and setting $d \mu$ in Theorem 2.1 in [3] to be $d E_{f, f}$, for a generic $f$, it is simpler to give the adapted proof. The second inequality given in the next theorem is a generalization of the result in 9], with a simpler proof given below. In the following, it will be assumed that products of index functions are defined pointwise; i.e.,

$$
(\phi \theta)(\lambda)=\phi(\lambda) \theta(\lambda) .
$$

Theorem 1. Let $\phi, \psi$ and $\theta$ be three index functions and $\Phi$ and $\Psi$ concave functions $(0, \infty) \rightarrow(0, \infty)$.

- (Generalized Hölder Inequality) If

$$
1 \leq \Phi(\phi(\lambda)) \Psi(\psi(\lambda)) \quad \text { a.e. }
$$

then

$$
1 \leq \Phi\left(\|f\|_{\phi \theta}^{2} /\|f\|_{\theta}^{2}\right) \Psi\left(\|f\|_{\psi \theta}^{2} /\|f\|_{\theta}^{2}\right)
$$

for all $f \in H_{(\phi+\psi+1) \theta}$.

- (Variable Hilbert Scale Inequality) If

$$
\phi(\lambda) \leq \Psi(\psi(\lambda)) \quad \text { a.e. }
$$

then

$$
\|f\|_{\phi \theta}^{2} /\|f\|_{\theta}^{2} \leq \Psi\left(\|f\|_{\psi \theta}^{2} /\|f\|_{\theta}^{2}\right)
$$

for all $f \in H_{(\phi+\psi+1) \theta}$.

Proof. In the following, let the measure $\nu$ be defined by $d \nu(\lambda)=\|f\|_{\theta}^{-2} \theta(\lambda) d E_{f, f}(\lambda)$ for $f \in H_{(\phi+\psi+1) \theta}$. By definition, because $\theta$ is positive and the integral of $d \nu$ equals $1, \nu$ is a probability measure.

From the inequality (마), one obtains

$$
1=\int_{0}^{\infty} d \nu(\lambda) \leq \int_{0}^{\infty} \Phi^{1 / 2}(\phi(\lambda)) \Psi^{1 / 2}(\psi(\lambda)) d \nu(\lambda) .
$$

After taking the square of the right-hand side, an application of the Cauchy-Schwarz inequality yields

$$
1 \leq \int_{0}^{\infty} \Phi(\phi(\lambda)) d \nu(\lambda) \int_{0}^{\infty} \Psi(\psi(\lambda)) d \nu(\lambda) .
$$

Since both $\Phi$ and $\Psi$ are concave, one uses the (inverse) Jensen inequality to obtain

$$
1 \leq \Phi\left(\int_{0}^{\infty} \phi(\lambda) d \nu(\lambda)\right) \Psi\left(\int_{0}^{\infty} \psi(\lambda) d \nu(\lambda)\right) .
$$

The required inequality (7) is then obtained by replacing $d \nu(\lambda)$ by its definition.

From the inequality (8) one obtains

$$
\int_{0}^{\infty} \phi(\lambda) d \nu(\lambda) \leq \int_{0}^{\infty} \Psi(\psi(\lambda)) d \nu(\lambda) \leq \Psi\left(\int_{0}^{\infty} \psi(\lambda) d \nu(\lambda)\right)
$$

using again the inverse Jensen inequality. The required inequality (9) is then obtained by replacing $d \nu(\lambda)$ by its definition. 
On choosing $\phi(\lambda)=\lambda^{-m}, \psi(\lambda)=\lambda^{m}$ and $\theta(\lambda)=1$ and $\Phi(\lambda)=\Psi(\lambda)=\lambda$ in the generalized Hölder inequality, a special case of a generalized Hölder inequality which holds for Sobolev spaces (see [1, p. 50]) is recovered; namely,

$$
\|f\|^{2} \leq\|f\|_{-m}\|f\|_{m}
$$

where $\|f\|_{-m}:=\|f\|_{\phi}$ and $\|f\|_{m}:=\|f\|_{\psi}$.

The (original) interpolation inequality for variable Hilbert scales (see Theorem 2.2 in [9]) can now be obtained from Theorem 1 by choosing $\Psi=\phi^{-1} \circ \psi$.

Corollary 1 (Interpolation inequality [9]). Let $\phi, \psi$ and $\theta$ be index functions.

- If $\phi \circ \psi^{-1}$ is concave, then

$$
\|f\|_{\phi \theta}^{2} \leq\|f\|_{\theta}^{2} \phi \circ \psi^{-1}\left(\|f\|_{\psi \theta}^{2} /\|f\|_{\theta}^{2}\right), \quad f \in H_{(\phi+\psi+1) \theta}, \quad f \neq 0 .
$$

- If $\phi$ and $\psi$ are strictly increasing and $\phi \circ \psi^{-1}$ is concave, then

$$
\phi^{-1}\left(\frac{\|f\|_{\phi \theta}^{2}}{\|f\|_{\theta}^{2}}\right) \leq \psi^{-1}\left(\frac{\|f\|_{\psi \theta}^{2}}{\|f\|_{\theta}^{2}}\right), \quad f \in H_{(\phi+\psi+1) \theta} \quad f \neq 0 .
$$

Proof. Choosing $\Psi(\lambda)=\phi \circ \psi^{-1}(\lambda)$, it follows that $\phi(\lambda)=\Psi(\psi(\lambda))$. Furthermore, $\Psi$ is concave. The following inequality then follows from Theorem 1

$$
\frac{\|f\|_{\phi \theta}}{\|f\|_{\theta}^{2}} \leq \phi\left(\psi^{-1}\left(\|f\|_{\psi \theta}^{2} /\|f\|_{\theta}\right)\right)
$$

which can be rearranged to give the first inequality. The second inequality follows from the monotonicity of $\phi^{-1}$.

We now recover the (ordinary) Hilbert scale inequalities from both inequalities of Theorem 1. First choose $\theta(\lambda)=1, \phi(\lambda)=\lambda^{-r}, \psi(\lambda)=\lambda^{s}, \Phi(\lambda)=\lambda^{s /(s+r)}$ and $\Psi(\lambda)=\lambda^{r /(s+r)}$ for some $s, r>0$. Consequently, $\Phi$ and $\Psi$ are both concave and, furthermore,

$$
\Phi(\phi(\lambda)) \Psi(\psi(\lambda))=\lambda^{-r s /(s+r)} \lambda^{s r /(s+r)}=1 .
$$

Hence, the conditions for the generalised Hölder inequality are fulfilled and one gets from Theorem 1 the inequality

$$
1 \leq\left(\frac{\|f\|_{-r}^{2}}{\|f\|^{2}}\right)^{s /(s+r)}\left(\frac{\|f\|_{s}^{2}}{\|f\|^{2}}\right)^{r /(s+r)}
$$

where $\|\cdot\|_{-r}=\|\cdot\|_{\phi}$ and $\|\cdot\|_{s}=\|\cdot\|_{\psi}$. From this, one obtains the classical bound

$$
\|f\| \leq\|f\|_{-r}^{s /(s+r)}\|f\|_{s}^{r /(s+r)},
$$

which is a special case of the standard interpolatory inequality (11). In order to get this bound one needed to "solve" the inequality for $\|f\|$, which is possible for this special case but not in general. This is one motivation for the variable Hilbert scale inequality. In order to recover the classical inequality from inequality (9), one chooses $\theta(\lambda)=\lambda^{-r}, \phi(\lambda)=\lambda^{r}, \psi(\lambda)=\lambda^{s+r}$, and $\Psi(\lambda)=\phi\left(\psi^{-1}(\lambda)\right)=\lambda^{r /(s+r)}$. Then inequality (9) becomes

$$
\frac{\|f\|^{2}}{\|f\|_{-r}^{2}} \leq\left(\frac{\|f\|_{s}^{2}}{\|f\|_{-r}^{2}}\right)^{r /(s+r)}
$$

and a simple multiplication of both sides with $\|f\|_{-r}^{2}$ gives the classical bound (12).

The variable Hilbert scales are used to derive error bounds for the regularization techniques for $A f=u$ given $u_{\delta}$ for which $\left\|u_{\delta}-u\right\| \leq \delta$. These error bounds rely on 
source conditions $f \in R(B)$ for some operator $B$. For the OHS, one requires that $B$ commutes with $A^{*} A$, i.e., $B=\psi\left(A^{*} A\right)$. In the classical theory, one requires that the source condition holds for some $B=\left(A^{*} A\right)^{s}$.

2.1. Scales of dilational interpolatory inequalities (DII). Two different classes of dilational interpolatory inequalities are introduced, as they lead to different types of error analysis for deconvolutional peak sharpening.

2.1.1. Positive index dilational interpolatory inequalities (PIDII). A new special family of variable Hilbert scales can be generated for a monotonically increasing index function $q:[0, \infty) \rightarrow[0, \infty)$ with $q(0)=0$, when the index functions are all of the form $\phi(\lambda)=1+c q(s \lambda)$, with $s>0$ and $c>0$. The corresponding norms are defined to be

$$
\|f\|_{s}^{2}=\|f\|^{2}+c \int_{0}^{\infty} q(s \lambda) d E_{f f}(\lambda) .
$$

The Hilbert space with norm $\|\cdot\|_{s}$ will be denoted by $H_{s}$. As the index functions are obtained from dilations of the original function $q(\lambda)$, we will refer to this family of variable Hilbert scales as dilational Hilbert scales (DHS). Because the generating index function $q$ is monotonically increasing, it follows that, for $s \leq t$ and $f \in H_{t}$, $\|f\|_{s} \leq\|f\|_{t}$, which implies the existence of a continuous embedding $H_{s} \hookrightarrow H_{t}$. Since $q(0)=0$, it follows that $\|f\|_{0}=\|f\|$ and $H_{0}=H$.

While there is no $a(\cdot)$ such that the DHS generated by $a(\cdot)$ and $T$ are equal to the OHS generated by $T$, the OHS can still be generated as a DHS.

Proposition 1. If $A$ is a positive definite operator and $T=e^{A}$, then the OHS generated by $T$ is equal to the DHS generated by $A$ with $a(\lambda)=e^{\lambda}$.

Proof. The proof shows that the norms $\|\cdot\|_{s}$ are the same in both cases. In the first case, the norm is

$$
\|f\|_{s}:=\int_{0}^{\infty} \lambda^{s} d E_{f, f}(\lambda)
$$

where $d E_{f, f}(\lambda)$ is the spectral measure of $T$. The DHS generated by $A$ with $a(\lambda)=$ $e^{\lambda}$ has the norm

$$
\|f\|_{s}^{\#}:=\int_{0}^{\infty} e^{\mu s} d \widetilde{E}_{f, f}(\mu)
$$

where $d \widetilde{E}_{f, f}(\mu)$ is the spectral measure generated by $A$. A consequence of the spectral theorem for selfadjoint operators is $\widetilde{E}_{f, f}(\mu)=E_{f, f}\left(e^{\mu}\right)$. Consequently, the two Hilbert scales are the same.

It follows that the dilational Hilbert scale, generated by the positive definite operator $A$ with $a(\lambda)=\exp (\lambda)$, consists of spaces $H_{s}=R\left(e^{-s A}\right)$, where $R\left(e^{-t A}\right)$ is the range of the elements $e^{-t A}$ of the semigroup generated by $-A$. In the case of $A=-d^{2} / d t^{2}$, this is just the set of solutions of the heat equation at times $t$. For the dilational Hilbert scale, this recovers the classical error bounds for the solution of the (time) inverse heat equation.

For the construction of $q$, choose any non-decreasing integrable function

$$
\eta:[0, \infty) \rightarrow \mathbb{R},
$$

for which $\eta(\nu) / \nu$ is integrable, and let

$$
q(\lambda):=\int_{0}^{\lambda} \exp \left(\int_{1}^{\tau} \eta(\nu) \frac{d \nu}{\nu}\right) d \tau .
$$


Lemma 1. For $\eta:[0, \infty) \rightarrow \mathbb{R}$ with $\eta(\nu) / \nu$ integrable, the function $q(\lambda)$ of equation (13) is

(1) monotonically increasing and differentiable with an absolutely continuous derivative, and

(2) if $q \in C^{2}[0, \infty)$, then

$$
\frac{\ddot{q}(\lambda)}{\dot{q}(\lambda)}=\frac{\eta(\lambda)}{\lambda}
$$

Proof. The integrability condition guarantees that $\int_{0}^{1} \eta(\nu) / \nu d \nu$ is finite. The differentiability of $q$ is an immediate consequence of its definition in equation (13). Differentiation of (13) yields

$$
\dot{q}(\lambda)=\exp \left(\int_{1}^{\lambda} \frac{\eta(\nu)}{\nu} d \nu\right)
$$

which is absolutely continuous. With $q \in C^{2}[0, \infty)$, a second differentiation of (13) yields

$$
\ddot{q}=\frac{\eta(\lambda)}{\lambda} \exp \left(\int_{1}^{\lambda} \frac{\eta(\nu)}{\nu} d \nu\right) .
$$

Combining these last two equations gives the result in (2) of Lemma 1.

The next theorem identifies an important subclass of DHS which generate a quite special and useful set of interpolation inequalities.

Theorem 2 (Positive Index Dilational Interpolatory Inequality). Let

(1) $\eta:[1, \infty) \rightarrow \mathbb{R}_{+}$be increasing, continuous and such that $\eta(\nu) / \nu$ is integrable,

(2) $q(\lambda):=\int_{0}^{\lambda} \exp \left(\int_{1}^{\tau} \eta(\nu) \frac{d \nu}{\nu}\right) d \tau$,

(3) the function $a(\lambda)$ be defined by

$$
a(\lambda)=1+c q(\lambda),
$$

(4) $\|f\|_{s}^{2}:=\int_{0}^{\infty} a(s \lambda) d E_{f f}(\lambda)$

(5) $\sigma \in(0,1)$.

Then, the function $\Psi(\lambda)=a\left(\sigma a^{-1}(\lambda)\right)$ is concave and $H_{s}:=\left\{f \mid\|f\|_{s}<\infty\right\}$ defines a Hilbert scale with the dilational interpolation inequalities (PIDII)

$$
\|f\|_{\sigma}^{2} \leq\|f\|^{2} a\left(\sigma a^{-1}\left(\|f\|_{1}^{2} /\|f\|^{2}\right)\right) .
$$

Proof. Let $\phi, \psi$ and $\theta$ be such that

$$
\phi(\lambda)=a(\sigma \lambda), \quad \psi(\lambda)=a(\lambda), \quad \theta(\lambda)=1, \text { for } \lambda \in(0, \infty) .
$$

Then $\phi, \psi$ and $\theta:(0, \infty) \rightarrow(0, \infty)$. By Lemma 1, $\phi$ and $\psi$ are continuous and, hence, are index functions. Hence, $\left\{H_{s}\right\}$ defines a Hilbert scale. In addition, because, by Lemma 1, $\phi$ is monotonically increasing, it is invertible. Thus, $\chi:=$ $\psi \circ \phi^{-1}$ is well defined. The chain rule gives

$$
\dot{\chi}(\phi(\lambda))=\frac{\dot{\psi}(\lambda)}{\dot{\phi}(\lambda)}
$$


which, also on the basis of Lemma 1, is well defined because $\dot{\psi}=c \dot{q}(\lambda)>0$ and $\dot{\phi}=c \sigma \dot{q}(\sigma \lambda)>0$. A second application of the chain rule gives

$$
\ddot{\chi}(\phi(\lambda))=\frac{\ddot{\psi} \dot{\phi}-\dot{\psi} \ddot{\phi}}{\dot{\phi}^{3}}=\frac{\dot{\psi}}{\dot{\phi}^{2}}\left\{\frac{\ddot{\psi}}{\dot{\psi}}-\frac{\ddot{\phi}}{\dot{\phi}}\right\} .
$$

Returning again to Lemma 1 gives

$$
\frac{\ddot{\psi}}{\dot{\psi}}=\frac{\ddot{q}}{\dot{q}}=\frac{\eta(\lambda)}{\lambda}
$$

and

which implies that

$$
\frac{\ddot{\phi}}{\dot{\phi}}=\sigma \frac{\ddot{q}(\sigma \lambda)}{\dot{q}(\sigma \lambda)}=\frac{\eta(\sigma \lambda)}{\lambda}
$$

$$
\ddot{\chi}(\phi(\lambda))=\frac{\dot{\psi}}{\dot{\phi}^{2}}\left\{\frac{\eta(\lambda)-\eta(\sigma \lambda)}{\lambda}\right\} .
$$

Because $\dot{\chi}(\lambda)>0, \eta(\lambda)$ is increasing and $\lambda>0$, it follows that $\ddot{\chi}(\phi(\lambda))>0$. Thus, $\chi(\lambda)$ is convex and $\Psi=\phi \circ \psi^{-1}=\chi^{-1}$ is concave. It now follows from Corollary 1 , on taking account of item (4) in the theorem and using the fact that $\|f\|_{\theta}^{2}=\|f\|^{2}$ and $\|f\|_{\psi}^{2}=\|f\|_{1}^{2}$, that

$$
\|f\|_{\sigma}^{2}=\|f\|_{\phi}^{2} \leq\|f\|^{2} \phi \circ \psi^{-1}\left(\|f\|_{1}^{2} /\|f\|^{2}\right)
$$

which yields the interpolatory inequality (15).

The proof shows, in particular, that the concavity of $\Psi$ (which is the key condition in Theorem 1 for the application of Jensen's inequality) is a consequence of equations (13) and (14).

The simplest example arises on choosing $\eta(\nu)=\nu$ and $c=e$, which corresponds to taking $q(\lambda)=(\exp (\lambda)-1) / e$ and, hence, $a(\lambda)=\exp (\lambda)$. Another example, which is the limiting example for the convexity condition, arises when $\eta(\nu)$ is the constant $(\gamma-1)$ and $c=\gamma$, which corresponds to taking $q(\lambda)=\lambda^{\gamma}$ and, hence, $a(\lambda)=1+\lambda^{\gamma}$. Even though this generates a one-parameter family of scales like the OHS family, it is a different family because its index function takes the form $a(s \lambda)=1+s^{\gamma} \lambda^{\gamma}$ where $s$ is the parameter indexing the Hilbert spaces $H_{s}$.

2.1.2. Negative index dilational interpolatory inequalities (NIDII). For the analysis of peak sharpening, it turns out that the Hilbert scales based on index functions of the form

$$
\phi(\lambda)=\frac{1}{a(s \lambda)}
$$

(where $a$ is the same as in Theorem 2) are more useful. One first defines the norms

$$
\|f\|_{-s}^{2}:=\int_{0}^{\infty} \frac{d E_{f, f}(\lambda)}{a(s \lambda)}, \quad f \in L_{2}(\mathbb{R}) .
$$

In this way, the Hilbert scale $\left\{H_{s}\right\}$ is extended by adding $\left\{H_{-s}\right\}$, where each $H_{-s}$ (for $s>0$ ) is defined to be the topological closure of $L_{2}(\mathbb{R})$ with respect to $\|\cdot\|_{-s}$. It can then be shown, using the Cauchy-Schwarz inequality, that

$$
\|f\| \leq\|f\|_{s}\|f\|_{-s}, \quad f \in H_{s}, s>0,
$$


and, hence, that $H_{-s}$ corresponds to the dual space of $H_{s}$ and that $\left(H_{-s}, L_{2}(\mathbb{R}), H_{s}\right)$ forms a Gelfand triple. Most importantly for peak sharpening, the following dilational interpolation inequality is obtained.

Proposition 2 (Negative Index Dilational Interpolatory Inequalities). Let a be as in Theorem 2, $\sigma \in(0,1)$ and furthermore, let

$$
\Psi(\lambda):=\frac{\lambda}{a\left(\sigma a^{-1}(\lambda)\right)}
$$

be concave. Then, for the norm

$$
\|f\|_{-s}^{2}:=\int_{0}^{\infty} \frac{1}{a(s \lambda)} d E_{f f}(\lambda), \quad s>0, f \in L_{2}(\mathbb{R}),
$$

the following dilational interpolation inequality (NIDII) holds:

$$
\|f\|_{-\sigma}^{2} \leq \frac{\|f\|^{2}}{a\left(\sigma a^{-1}\left(\|f\|^{2} /\|f\|_{-1}^{2}\right)\right)}, \quad f \in L_{2}(\mathbb{R}) .
$$

Proof. Let $\phi, \psi$ and $\theta$ be such that

$$
\phi(\lambda)=a(\lambda) / a(\sigma \lambda), \quad \psi(\lambda)=a(\lambda), \quad \theta(\lambda)=1 / a(\lambda), \text { for } \lambda \in(0, \infty) .
$$

It then follows from Lemma 1 that $\phi, \psi$ and $\theta$ are continuous, and, hence, are index functions. By construction $\psi$ is invertible and, furthermore,

$$
\phi(\lambda)=\Psi(\psi(\lambda)) .
$$

As we assume that $\Psi$ is concave, we can invoke the interpolation inequality of Theorem 1 which is

$$
\frac{\|f\|_{-\sigma}^{2}}{\|f\|_{-1}^{2}} \leq \frac{\|f\|^{2} /\|f\|_{-1}^{2}}{a\left(\sigma a^{-1}\left(\|f\|^{2} /\|f\|_{-1}^{2}\right)\right)} .
$$

The required DII is then obtained by multiplication by $\|f\|_{-1}^{2}$.

Unlike in Theorem 2, the concise characterization of all the $\eta$, for which $\Psi$ turns out to be concave, is still an open question. However, for the Gaussian, Lorentzian, Voigt and exponential peaks, this will be verified for each, separately. As the concavity of $\Psi$ is essential, it is assumed to hold in Proposition 2. By definition, $\Psi$ maps $(0, \infty)$ into $(0, \infty)$ and one consequence of the assumed concavity is that $\Psi$ cannot be decreasing.

By way of contrast, conditions for the concavity of $\Phi(\lambda)=a\left(\sigma a^{-1}(\lambda)\right)$ have been established in Theorem 2. A natural question to ask is: when does the concavity of $\Phi$ imply the concavity of $\Psi(\lambda)=\lambda / \Phi(\lambda)$ ? While again we do not know the answer to this question, one observes that in the OHS case, where one has $\Phi(\lambda)=\lambda^{\sigma}$ for some $\sigma \in(0,1)$, it follows that $\Psi$ is concave. This is the most prominent case of an operator concave function $\Phi$ [4]. It would appear appropriate to investigate which properties of $\eta$ result in operator concave $\Phi$ which imply concavity of $\Psi$.

A larger class of applications is obtained when $\Psi$ is chosen to be concave with $\phi(\lambda) \leq \Psi(\psi(\lambda))$; in particular, when one chooses the smallest such $\Psi$; i.e., the concave majorant of $\phi \circ \psi^{-1}$. For such a concave majorant to exist, one only requires asymptotic concavity of $\Psi(\lambda)$. In the current context, because $\psi(\lambda)=a(\lambda)$ is monotonically increasing, this will hold if $\dot{\Psi}(\psi(\lambda))=\dot{\phi}(\lambda) / \dot{\psi}(\lambda)$ is asymptotically decreasing. 


\section{Application-Deconvolution By SHARPEning}

The theory discussed above provides a natural framework in which to analyze the sharpening (narrowing) of broadened and possibly overlapping spectroscopic peaks by deconvolution. Because, as shown in the introduction, the convolution of two Gaussian peaks is a Gaussian peak, an OHS analysis can be applied successfully when the peak and its broadening are both modeled as Gaussians. In the DHS framework, general dilational-parameterized peaks can be analyzed with similar facility. The VHS framework could be applied, but additional assumptions would have to be invoked, like the ones given above in Theorem 2 for DHS, before error estimates as sharp and useful as those given below could be derived. In essence, Theorem 2 generates a framework which allows the VHS methodology to be applied directly to deconvolution by sharpening (narrowing).

Spectroscopy reveals information about the chemical composition of samples and is an important tool in chemistry, physics, biology, astronomy and related industrial applications. The data consists of a superposition of "peaks". In the case of overlapping peaks, their separation and identification poses a substantial challenge. Methods for performing such tasks are discussed in [11. They have wide applicability and can also be used for deblurring in image processing.

The widening of the peaks in a spectrum results from a "diffusion" of information into neighboring frequencies. If this "diffusion" is independent of location, it can be modeled as a convolution. In an $L_{2}(\mathbb{R})$ Hilbert space context, the theoretical model takes the form given in equations (2) and (3).

Rather than attempting to accurately perform the full deconvolution, it is more sensible to "sharpen" the spectrum so that a better identifiability of the locations and number of peaks is achieved compared with that available from a visual inspection of the available data $u^{\delta}$ [2, 9]. As explained in the Introduction, this corresponds to finding the solution $z$ of the sharpening equation

$$
\tilde{B}_{\beta} * z=u
$$

for the data $u^{\delta}$ where the regularity of $z$ is determined by the source condition

$$
z=B_{\beta} * f, \quad f \in L_{2}(\mathbb{R}) .
$$

In order to utilize the DHS interpolation inequality framework developed above, it is necessary to introduce a DHS which guarantees that $z$ is in $H_{s}$. A general framework for doing this is given by

Theorem 3. Let the operator $T$ that generates the dilational Hilbert scale $\left\{H_{s}\right\}$ be $-d^{2} / d x^{2}$, and define the Fourier transform to take the form

$$
\hat{f}=\int_{\mathbb{R}} \exp (i \omega t) f(t) d t .
$$

If $s=\beta^{2}$, if the absolute value of the Fourier transform of the given peak function $b$ is symmetric with

$$
|\hat{b}(\omega)|=|\hat{b}(-\omega)|
$$

and if the generating function a of $\left\{H_{s}\right\}$ satisfies

$$
a(\lambda)=\frac{1}{|\hat{b}(\sqrt{\lambda})|^{2}},
$$


then

$$
\left\|b_{\beta} * f\right\|_{s}=\|f\|, \quad f \in L_{2}(\mathbb{R}) .
$$

Proof. Using the Fourier transform $\hat{f}$, one obtains

$$
\int_{0}^{\infty} \lambda d E_{f f}(\lambda)=\frac{1}{2 \pi} \int_{\mathbb{R}} \omega^{2}|\hat{f}(\omega)|^{2} d \omega=\frac{1}{2 \pi} \int_{0}^{\infty} \omega^{2}\left(|\hat{f}(\omega)|^{2}+|\hat{f}(-\omega)|^{2}\right) d \omega
$$

with $\lambda=\omega^{2}$ and $d E_{f f}(\lambda)=\frac{1}{2 \pi}\left(|\hat{f}(\omega)|^{2}+|\hat{f}(-\omega)|^{2}\right) d \omega$. The resulting DHS norm is given by

$$
\|f\|_{s}^{2}=\frac{1}{2 \pi} \int_{0}^{\infty} a\left(s \omega^{2}\right)\left(|\hat{f}(\omega)|^{2}+|\hat{f}(-\omega)|^{2}\right) d \omega .
$$

A straightforward application of the definition of $b_{\beta}(t)$ and the Fourier transform proves that $\hat{b}_{\beta}(\omega)=\hat{b}(\beta \omega)$. With this and the assumed symmetry of equation (17), one then obtains for the left-hand side of condition (19),

$$
\left\|b_{\beta} * f\right\|_{s}^{2}=\frac{1}{2 \pi} \int_{0}^{\infty} a\left(s \omega^{2}\right)|\hat{b}(\beta \omega)|^{2}\left(|\hat{f}(\omega)|^{2}+|\hat{f}(-\omega)|^{2}\right) d \omega .
$$

On recalling that $s=\beta^{2}$ and condition (18), the right-hand side of the last equation becomes

$$
\frac{1}{2 \pi} \int_{0}^{\infty}\left(|\hat{f}(\omega)|^{2}+|\hat{f}(-\omega)|^{2}\right) d \omega=\|f\|^{2},
$$

which proves the theorem.

Together, Theorems 2 and 3 yield a procedure for determining whether, for a given peak function $b(t)$, the DII of equation (15) can be applied. First one uses condition (18) to determine $a(\lambda)$ and then checks that it is a valid generating function for the DII of Theorem 2, This is done by showing that there exists an $\eta(\lambda)$ such that $a(\lambda)$ has the structure given in equations (14) and (13). For this, one uses, from Lemma 1 and the form of $a(\lambda)$ just derived, the relationships

$$
\frac{\eta(\lambda)}{\lambda}=\frac{\ddot{a}(\lambda)}{\dot{a}(\lambda)}, \quad c=\dot{a}(1)
$$

to determine the corresponding $\eta(\lambda)$ and the constant $c$ in equation (14). The process is validated and finalized on showing that, with respect to the assumptions of Lemma 1, the resulting $\eta(\lambda)$ is non-decreasing.

3.1. Error analysis for peak sharpening. Depending on the type of DII utilized, the error analysis of peak sharpening can take two quite different forms. Here, let $r$ denote the residual associated with the error $e=\left(z-z_{\alpha}^{\delta}\right)$ with $z_{\alpha}^{\delta}$ denoting the regularized solution of $\tilde{B}_{\beta} z=u^{\delta}$ by a regularization method with regularization parameter $\alpha$.

3.1.1. Error analysis using positive index dilational interpolatory inequalities. The first step is the identification of conditions which guarantees that

$$
\|e\| \leq\|r\|_{\sigma}, \quad \sigma<1,
$$

since the DII of equation (15) then yields an upper bound for the right-hand side of (21), and, hence, for $\|e\|$. Here, by utilizing the results of Theorem 3, a Fourier transform constraint is derived that guarantees (21). From equation (4), it follows 
that there exists a $v$ such that $r=\tilde{B}_{\beta} e$ with $e=B_{\beta} v$. Consequently, $r=B v$ and the last inequality becomes

$$
\left\|B_{\beta} v\right\|=\|e\| \leq\|r\|_{\sigma}=\|B v\|_{\sigma}
$$

or, equivalently,

$$
\left\|b_{\beta} * v\right\| \leq\|b * v\|_{\sigma}, \quad \text { for all } v \in H .
$$

As the norm is a continuous function, it suffices to show that this bound holds for a dense subset in $L_{2}(\mathbb{R})$. Using equation (20), this last inequality can be rewritten as

$$
\frac{1}{2 \pi} \int_{0}^{\infty}\left[|\hat{b}(\beta \omega)|^{2}-a\left(\sigma \omega^{2}\right)|\hat{b}(\omega)|^{2}\right]\left(|\hat{v}(\omega)|^{2}+|\hat{v}(-\omega)|^{2}\right) d \omega \leq 0, \quad \text { a.e. }
$$

This relationship holds for all choices of $v$ in a dense subset of $L_{2}(\mathbb{R})$. On taking account of the connection (19) between $a(\lambda)$ and $\hat{b}(\sqrt{\lambda})$, it follows that

$$
|\hat{b}(\beta \omega)|^{2} \leq \frac{|\hat{b}(\omega)|^{2}}{|\hat{b}(\sqrt{\sigma} \omega)|^{2}}, \quad \omega \in \mathbb{R}, \quad \text { a.e. }
$$

or, equivalently,

$$
a(\lambda) \leq a(\sigma \lambda) a\left(\beta^{2} \lambda\right) .
$$

This is the "peak-constraint" that must be satisfied in order to guarantee the validity of the error estimate (21). Consequently, the form taken by the PIDII for the Gaussian, Lorentzian, Voigt and exponential peaks, depends on the value of $\sigma$ that results in guaranteeing the validity of this peak-constraint.

A bound on this error is given in the following corollary of Theorem 2 ,

Corollary 2. Let $v=f_{\epsilon}-f, r=B v$ and $e=B_{\beta} v$. Let $a(\lambda)=1 /|\hat{b}(\sqrt{\lambda})|^{2}$ satisfy the conditions of Theorem 2 , and let $b$ satisfy the peak constraint

$$
|\hat{b}(\beta \omega)| \cdot|\hat{b}(\sqrt{\sigma} \omega)| \leq|\hat{b}(\omega)|
$$

for some $\sigma \in(0,1)$. Then,

$$
\|e\| \leq\|r\| \sqrt{a\left(\sigma a^{-1}\left(\|v\|^{2} /\|r\|^{2}\right)\right)}
$$

Proof. Since $\|v\|=\|r\|_{1}$ and, by the peak constraint, $\|e\| \leq\|r\|_{\sigma}$, the bound follows directly from the PIDII of Theorem 2 .

3.1.2. Error analysis using negative index dilational interpolatory inequalities. Consider the original reconstruction problem of finding the solution of the convolution equation $B f=u$ from data $u^{\delta}$ where $\left\|u^{\delta}-u\right\| \leq \delta$. Many regularization methods, (including Morozov's discrepancy principle [5]), construct a regularized solution $f_{\delta}$ which is consistent, in that it has a residual $r=B\left(f_{\delta}-f\right)$ that satisfied $\|r\| \leq C_{1} \delta$, and is stable with the error $v=f_{\delta}-f$ bounded, i.e., $\|v\| \leq C_{2}$. Unfortunately, without a source condition (e.g., $f \in \operatorname{Range}\left(\psi\left(B^{*} B\right)\right.$ ) for some index function $\psi$ ), one cannot apply the interpolation inequalities to get an error bound of the type $\|v\| \leq o(\epsilon)$. However, one can get a bound for the mollified solution $z_{\beta, \delta}=B_{\beta} f_{\delta}$ [10. This mollified solution is an approximation of the sharpened spectrum $B_{\beta} f$ and its error is $e=B_{\beta}\left(f_{\delta}-f\right)$. A bound on this error is given in the following corollary of Proposition 2 . 
Corollary 3. Let $v=f_{\epsilon}-f, r=B v$ and $e=B_{\beta} v$. Let $a(\lambda)=1 /|\hat{b}(\sqrt{\lambda})|^{2}$ satisfy the conditions of Theorem 2, and $\sigma=\beta^{2}$. Then,

$$
\|e\| \leq \frac{\|v\|}{\sqrt{a\left(\sigma a^{-1}\left(\|v\|^{2} /\|r\|^{2}\right)\right)}} .
$$

Proof. Since $\|r\|=\|B v\|=\|v\|_{-1}$ and $\|e\|=\left\|B_{\beta} v\right\|=\|v\|_{-\sigma}$, the bound follows directly from the NIDII of Proposition 3.

\subsection{The corresponding properties for the Gaussian, Lorentzian, Voigt} and exponential peaks. We now have the tools to examine and compare, using the DII given in equations (15) and (16), the sharpening (narrowing) resulting from deconvolution with the Gaussian, Lorentzian, Voigt and exponential peaks.

For the proof of the concavity of $\phi \circ \psi^{-1}$ for the PIDII, it follows from Theorem 2 that it is only necessary to show that $\eta(\lambda)$ is non-decreasing. For the NIDII, once it is established that $a(\lambda)$ is monotonically increasing, it is only necessary to show that $\dot{\phi}(\lambda) / \dot{\psi}(\lambda)$ is decreasing, where $\phi(\lambda)=a(\lambda) / a(\sigma \lambda)$ and $\psi(\lambda)=a(\lambda)$.

3.2.1. The Gaussian peak. For $b_{G}(t)=\frac{1}{\sqrt{2 \pi}} \exp \left(-t^{2} / 2\right)$, following the above protocols, one first obtains

$$
\hat{b}_{G}(t)=\exp \left(-\omega^{2} / 2\right), \quad a_{G}(\lambda)=\exp (\lambda), \quad \eta_{G}(\lambda)=\lambda, \quad c_{G}=e .
$$

Here, $a_{G}^{-1}(\zeta)=\ln \zeta$. The non-decreasing of $\eta_{G}(\lambda)$ is immediately apparent. Equality holds for the peak-constraint (22) if $\sigma+\beta^{2}=1$. Concavity for the NIDII follows on noting that $\dot{\phi}(\lambda) / \dot{\psi}(\lambda)=(1-\sigma) \exp (-\sigma \lambda)$. Both the PIDII and the NIDII yield

$$
\|e\| \leq\|v\|^{(1-\sigma)}\|r\|^{\sigma}, \quad \sigma=1-\beta^{2} .
$$

3.2.2. The Lorentzian peak. For $b_{L}(t)=\frac{1}{\pi} \frac{1}{1+t^{2}}$, following the above protocols, one first obtains

$$
\hat{b}_{L}(t)=\exp (-|\omega|), \quad a_{L}(\lambda)=\exp (2 \sqrt{\lambda}), \quad \eta_{L}(\lambda)=\sqrt{\lambda}-\frac{1}{2}, \quad c_{L}=e^{2} .
$$

Here, $a_{G}^{-1}(\zeta)=(\ln (\zeta) / 2)^{2}$. The non-decreasing of $\eta_{L}(\lambda)$ is immediately apparent. The peak-constraint is satisfied if $\sigma+\beta^{2}=1$. Concavity for the NIDII follows on noting that $\dot{\phi}(\lambda) / \dot{\psi}(\lambda)=(1-\sqrt{\sigma}) \exp (-2 \sqrt{\sigma \lambda})$. Both the PIDII and the NIDII yield

$$
\|e\| \leq\|v\|^{1-\sqrt{\sigma}}\|r\|^{\sqrt{\sigma}}, \quad \sigma=1-\beta^{2} .
$$

They have the same form as for the Gaussian except that the $\sigma$ of the Gaussian has become $\sqrt{\sigma}$.

3.2.3. The Voigt peak. For $b_{V}(t)=\int_{-\infty}^{\infty} b_{G}(t-\tau) b_{L}(\mu \tau) d \tau$, following the above protocols, one first obtains

$$
\begin{gathered}
\hat{b}_{V}(t)=\exp \left(-\left(\omega^{2}+\mu|\omega|\right)\right), \quad a_{V}(\lambda)=\exp (\lambda+2 \mu \sqrt{\lambda}), \\
\eta_{V}(\lambda)=(\lambda+\mu \sqrt{\lambda})-\frac{1}{2} \frac{\mu}{(\sqrt{\lambda}+\mu)}, \quad c_{V}=(1+\mu) \exp (1+2 \mu) .
\end{gathered}
$$

Proposition 3. Let $r, v, e$ be as in Corollary 3 and let $a(\lambda)=\exp (\lambda+\mu \sqrt{\lambda})$ for some $\mu \in[0,2]$. Furthermore, let $\|r\| /\|v\| \leq \epsilon \leq 1$ and

$$
\theta=\sigma+\frac{\mu(1-\sqrt{\sigma}) \sqrt{\sigma}}{(1+\mu /(2|\log (\epsilon)|))}
$$


for some $\sigma \in(0,1)$. Then

$$
\|e\| \leq\|v\|^{1-\theta}\|r\|^{\theta} .
$$

Remark. As illustrated in the proof, the negative norm results of Proposition 2 play the key role. It represents a result that cannot be obtained using the positive norms.

Proof. We directly apply Theorem 1 as in Proposition 2. In particular, we choose $\Psi(\lambda)=\lambda^{1-\theta}$. We then have to show that $\Psi$ is concave and that

$$
\Psi(\lambda) \geq \lambda / a\left(\sigma a^{-1}(\lambda)\right)
$$

for large enough $\lambda$.

Clearly, $\Psi$ is concave if and only if $\theta \in[0,1]$. As $\sigma \in(0,1)$, it follows that $\theta \geq 0$. Then, because $\mu \leq 2$, one has

$$
\begin{aligned}
\theta & \leq \sigma+\mu(1-\sqrt{\sigma}) \sqrt{\sigma} \\
& \leq \sigma+2(1-\sqrt{\sigma}) \sqrt{\sigma} \\
& =1-(1-\sqrt{\sigma})^{2} \leq 1 .
\end{aligned}
$$

So concavity is established and the remainder of the proof establishes that $\Psi(\lambda) \geq$ $\lambda / a\left(\sigma a^{-1}(\lambda)\right)$ for $\lambda \geq 1 / \epsilon^{2}$. On observing that $a(\lambda)=\exp (\alpha(\sqrt{\lambda}))$ for $\alpha(\eta)=$ $\eta^{2}+\mu \eta$, it follows that $a^{-1}(\eta)=\alpha^{-1}(\log (\eta))^{2}$ and, hence,

$$
a\left(\sigma a^{-1}(\lambda)\right)=\exp \left(\alpha\left(\sqrt{\sigma} \alpha^{-1}(\log (\lambda))\right)\right) .
$$

It is clear that, on using a consistency argument,

$$
\alpha^{-1}(\xi)=-\frac{\mu}{2}+\sqrt{\frac{\mu}{2}+\xi} .
$$

As $\xi=\log (\lambda) \geq 2|\log (\epsilon)|$, it follows that

$$
\begin{aligned}
\alpha\left(\sqrt{\sigma} \alpha^{-1}(\xi)\right) & =\sigma \xi+\mu(1-\sqrt{\sigma}) \sqrt{\sigma}\left(\sqrt{\xi+\mu^{2} / 4}-\mu / 2\right) \\
& =\xi\left(\sigma+\mu(1-\sqrt{\sigma}) \sqrt{\sigma}\left(\sqrt{1+(\mu /(2 \xi))^{2}}-\mu /(2 \xi)\right)\right) \\
& =\xi\left(\sigma+\frac{\mu(1-\sqrt{\sigma}) \sqrt{\sigma}}{\sqrt{1+(\mu /(2 \xi))^{2}}+\mu /(2 \xi)}\right) \\
& \geq \xi\left(\sigma+\frac{\mu(1-\sqrt{\sigma}) \sqrt{\sigma}}{1+\frac{\mu}{\xi}}\right) \\
& \geq \xi\left(\sigma+\frac{\mu(1-\sqrt{\sigma}) \sqrt{\sigma}}{1+\frac{\mu}{2|\log (\epsilon)|}}\right)=\xi \theta .
\end{aligned}
$$

Consequently, one has $a\left(\sigma a^{-1}(\lambda)\right)=\exp \left(\alpha\left(\sqrt{\sigma} \alpha^{-1}(\log (\lambda))\right)\right) \geq \lambda^{\theta}$ and so

$$
\Psi(\lambda)=\lambda^{1-\theta} \geq \frac{\lambda}{a\left(\sigma a^{-1}(\lambda)\right)}, \quad \text { for } \lambda \geq \epsilon^{-2} .
$$


3.2.4. The exponential peak. For $b_{E}(t)=\exp (-|t|) / 2$, following the above protocols, one first obtains

$$
\hat{b}_{E}(t)=\frac{1}{1+\omega^{2}}, \quad a_{E}(\lambda)=(1+\lambda)^{2}, \quad \eta_{E}(\lambda)=\frac{2 \lambda}{1+\lambda}, \quad c_{E}=4 .
$$

Here, $a_{E}^{-1}(\zeta)=\zeta^{1 / 2}-1$. As $\lambda$ increases, $\eta_{E}(\lambda)$ increases. The peak-constraint is satisfied if $\sigma+\beta^{2}=1$. Consequently, the PIDII becomes

$$
\|e\| \leq(1-\sigma)\|v\|+\sigma\|r\|, \quad \sigma=1-\beta^{2} .
$$

Concavity for the NIDII follows on noting that $\dot{\phi}(\lambda) / \dot{\psi}(\lambda)=\frac{1-\sigma}{(\sigma \lambda+1)^{3}}$. Consequently, the NIDII becomes

$$
\|e\| \leq \frac{\|v\|\|r\|}{\sigma\|v\|+(1-\sigma)\|r\|}=\frac{1}{\frac{\sigma}{\|r\|}+\frac{(1-\sigma)}{\|v\|}}, \quad \sigma=1-\beta^{2} .
$$

This gives a clear illustration that there exist situations where the structure of the NIDII will be quite different from that of the PIDII. The importance of this NIDII in characterizing the special behavior of the exponential peak has already been explained, in terms of the structure of equation (5) in the Introduction.

\section{Conclusions}

We have shown how a new class of Hilbert scales based on dilations leads to error bounds for numerical peak sharpening procedures. Specifically, these bounds are derived from a new interpolation inequality of these Hilbert scales. Specific bounds were found for Gaussian, Lorentz, Voigt and exponential peaks. In the case of the Gaussian and Lorentz peaks, the same bounds can also be obtained from classical interpolation inequalities. The bounds for Voigt and exponential peaks, however, require the new theory.

These error bounds quantify the fundamental trade-off of peak sharpening: Narrower peaks, i.e., finer resolution, come at a cost of larger errors. The noise in the data thus limits fundamentally the resolution achievable by computational narrowing procedures. Low data errors can now be achieved in modern spectroscopic measurements (see, for example, the NIR spectra in 23]). This then leads to higher resolution and the improvement in resolution, achieved from the better data, can be analyzed using our new error bounds.

While we have provided a general theory, the analysis of specific narrowing procedures, based, e.g., on mollification or Tikhonov regularization, has not been done in detail here. Furthermore, there is an open question regarding the characterization in Proposition 2 of the asymptotic concavity of $\Psi(\lambda)=\lambda / a\left(\sigma a^{-1}(\lambda)\right)$ in terms of the function $\eta(\nu)$ of equation (13).

A basic assumption made in all of the discussion here is that the shape of the peaks does not depend on the frequency. In some applications, however, this does not hold. In this case, a different framework, than the one used here which is based on convolutions, is required.

While we have considered the four most important peaks, it would be of some interest to obtain bounds for other peaks as well, including peaks like the sinc function $b(t)=\sin (t) / t$. A challenge here is that one looses the monotonicity. Other peaks of interest could include derivatives of the four classical peaks discussed above. 
Interestingly, the DII for the Gauss, Lorentz and Voigt peaks, all have the same basic form,

$$
\|e\| \leq\|v\|^{(1-\theta)}\|r\|^{\theta},
$$

as the classical DII of equation (11). Consequently, the DII of equation (1) has been extended to a wider class of situations for a larger class of source conditions. This leaves open the question about the form that the source conditions must take to guarantee that the upper bound takes the form $O\left(\delta^{\theta}\right)$.

Finally, possibly the most exciting open question is how to analyze peak narrowing procedures based on non-linear regularization procedures like the ones studied in [19, 6].

\section{ACKNOWLEDGEMENTS}

Both authors wish to acknowledge with grateful thanks the suggestions of an unnamed referee which led to a considerable improvement in the paper's presentation and results. They also wish to acknowledge the support of the Radon Institute of Computational and Applied Mathematics, where the initial draft of this paper was finalized.

\section{REFERENCES}

[1] Robert A. Adams, Sobolev spaces, Academic Press [A subsidiary of Harcourt Brace Jovanovich, Publishers], New York, London, 1975, Pure and Applied Mathematics, Vol. 65. MR0450957(56:9247)

[2] Leland C. Allen, H. M. Gladney, and S. H. Glarum, Resolution enhancement for spectra of chemical and physical interest, The Journal of Chemical Physics 40 (1964), no. 11, 3135-3141.

[3] Pascal Bégout and Fernando Soria, A generalized interpolation inequality and its application to the stabilization of damped equations, J. Differential Equations 240 (2007), no. 2, 324-356. MR2351180 (2009a:35150)

[4] Rajendra Bhatia, Matrix analysis, Graduate Texts in Mathematics, vol. 169, Springer-Verlag, New York, 1997. MR1477662 (98i:15003)

[5] Heinz W. Engl, Martin Hanke, and Andreas Neubauer, Regularization of inverse problems, Mathematics and its Applications, vol. 375, Kluwer Academic Publishers Group, Dordrecht, 1996. MR1408680 (97k:65145)

[6] Markus Grasmair, Markus Haltmeier, and Otmar Scherzer, Sparse regularization with lq penalty term, Inverse Problems 24 (2008), no. 5, 055020 (13pp). MR2438955 (2010a:65086)

[7] Charles W. Groetsch, The theory of Tikhonov regularization for Fredholm equations of the first kind, Research Notes in Mathematics, vol. 105, Pitman (Advanced Publishing Program), Boston, MA, 1984. MR742928 (85k:45020)

[8] Markus Hegland, An optimal order regularization method which does not use additional smoothness assumptions, SIAM J. Numer. Anal. 29 (1992), no. 5, 1446-1461. MR.1182739 (93j:65090)

[9] - Variable Hilbert scales and their interpolation inequalities with applications to Tikhonov regularization, Appl. Anal. 59 (1995), no. 1-4, 207-223. MR.1378036 (97a:65060)

[10] Markus Hegland and Robert S. Anderssen, A mollification framework for improperly posed problems, Numer. Math. 78 (1998), no. 4, 549-575. MR.1606320 (99d:65190)

[11] Resolution enhancement of spectra using differentiation, Inverse Problems 21 (2005), no. 3, 915-934. MR.2144811 (2006a:65196)

[12] Selim G. Kreĭn and Yu. I. Petunin, Scales of Banach spaces, Uspehi Mat. Nauk 21 (1966), no. 2 (128), 89-168. MR0193499 (33:1719)

[13] Peter Mathé and Sergei V. Pereverzev, Geometry of linear ill-posed problems in variable Hilbert scales, Inverse Problems 19 (2003), no. 3, 789-803. MR1984890 (2004i:47021)

[14] _ Regularization of some linear ill-posed problems with discretized random noisy data, Math. Comp. 75 (2006), no. 256, 1913-1929 (electronic). MR.2240642

[15] Peter Mathé and Ulrich Tautenhahn, Interpolation in variable Hilbert scales with application to inverse problems, Inverse Problems 22 (2006), no. 6, 2271-2297. MR.2277542 
[16] M. Thamban Nair, Sergei V. Pereverzev, and U. Tautenhahn, Regularization in Hilbert scales under general smoothing conditions, Inverse Problems 21 (2005), no. 6, 1851-1869. MR2183654 (2006k:47026)

[17] Frank Natterer, Error bounds for Tikhonov regularization in Hilbert scales, Applicable Anal. 18 (1984), no. 1-2, 29-37. MR762862 (86e:65081)

[18] J.J. Olivero and R.L. Longbothum, Empirical fits to the Voigt line width: A brief review, Journal of Quantitative Spectroscopy and Radiative Transfer 17 (1977), no. 2, 233-236.

[19] Ronny Ramlau and Gerd Teschke, A Tikhonov-based projection iteration for nonlinear illposed problems with sparsity constraints, Numerische Mathematik 104 (2006), no. 2, 177-203. MR.2242613(2007e:65057)

[20] Walter Rudin, Functional analysis, McGraw-Hill Book Co., New York, 1973, McGraw-Hill Series in Higher Mathematics. MR0365062 (51:1315)

[21] Torsten Schröter and Ulrich Tautenhahn, Error estimates for Tikhonov regularization in Hilbert scales, Numer. Funct. Anal. Optim. 15 (1994), no. 1-2, 155-168. MR1261605 (95c:65091)

[22] Ulrich Tautenhahn, Error estimates for regularization methods in Hilbert scales, SIAM J. Numer. Anal. 33 (1996), no. 6, 2120-2130. MR1427456 (97k:65148)

[23] Paul R. Wiley, Greg J. Tanner, Peter M. Chandler, and Robert S. Anderssen, Molecular Classification of Barley (Hordeum vulgare L.) Mutants Using Derivative NIR Spectroscopy, Journal of Agricultural and Food Chemistry 57 (2009), no. 10, 4042.

Centre for Mathematics and its Applications, The Australian National University, Canberra, ACT 0200, Australia

E-mail address: markus.hegland@anu.edu.au

CSiro Mathematics, Informatics and Statistics, GPO Box 664, Canberra, ACT 2601, Australia

E-mail address: bob.anderssen@csiro.au 